\title{
The Chebyshev-Galerkin method of Nonlinear Burgers equation
}

\author{
Lizheng Cheng ${ }^{1, ~ a}$, Hongping $\mathrm{Li}^{2,}$ b \\ ${ }^{1}$ School of information Science and Engineering, Hunan International Economics University, \\ Changsha, 410205, China; \\ ${ }^{2}$ Corresponding author: School of information Science and Engineering, Hunan International \\ Economics University, Changsha, 410205, China. \\ a243073049@qq.com, b443638675@qq.com
}

Keywords: Burgers equation, Chebyshev-Galerkin method, Crank-Nicolson Leap- frog scheme.

\begin{abstract}
The nonlinear Burgers equation is a hot issue in the field of computational fluid dynamics, which has nonlinear convection term and diffusion term. In this paper, we give a numerical solution of the nonlinear Burgers equation by a combination of finite difference and Chebyshev-Galerkin method. Use the Crank-Nicolson Leap-frog scheme in time, and the Chebyshev-Galerkin method in space to solve the nonlinear Burgers equation. Finally, numerical experiments are carried out to illustrate the efficiency of the proposed method.
\end{abstract}

\section{Introduction}

Burgers equation can be used as a mathematical model to describe many physical phenomena, in particular, it can be used as a mathematical model of fluid flow phenomena. It is a very important and basic partial differential equation in fluid mechanics, which is widely used in aerodynamics, turbulent flow, heat transfer, transportation flow, groundwater pollution and other fields. It has some properties of the Navier-Stokes equation, which can be regarded as a simple Navier-Stokes equation. And can be used to study the properties of the Navier-Stokes equation by numerical simulation. It usually produces shock waves in this equation, which brings some difficulties to the numerical solution. So, the further research on it will help us solve other nonlinear problems.

At the same time, the exact smooth solution of the Burgers equation can be constructed. Therefore, many nonlinear Burgers equation can be solved numerically by means of model which can be numerically tested. As result, it is important to study the efficient numerical method for the Burgers equation. The numerical approaches of the Burgers equation have been developed as follows: the finite element method,finite difference method and Chebyshev spectral method and spectral collocation method[1-4]. In 1915, Bateman firstly gave the stable solution of one dimensional viscous Burgers equation[5]. In some special initial boundary value condition, the exact solution of the Burgers equation can be arrived, but these exact solutions are usually in the form of infinite series. Therefore, we must calculate much more items to get the high accuracy, and the calculation process is also very complex.

The paper is organized as follows. Section 1 mainly introduces the physical back ground of the Burgers equation and the main numerical methods. Section 2 mainly, illustrates the basic principle of the Chebyshev-Galerkin method of the nonlinear Burgers equation. In section 3, we design the numerical scheme and algorithm of the nonlinear Burgers equation . The numerical experiments of the nonlinear Burgers equation are presented in section 4 to verify the efficiency of the method.

\section{Chebyshev-Galerkin method}

Let $\Omega=(-1,1)$ is an open set,we consider the following nonlinear Burgers equation with initial and bound conditions : 


$$
\left\{\begin{array}{l}
\frac{\partial u}{\partial t}=\varepsilon \frac{\partial^{2} u}{\partial x^{2}}-u \frac{\partial u}{\partial x}, \quad-1<x<1,0<t \leq T \\
u(0, t)=u(1, t)=0, \quad t>0, \\
u(x, 0)=u_{0}(x), \quad-1<x<1 .
\end{array}\right.
$$

where $\mathrm{u}$ is the speed, $\varepsilon$ is the viscosity constant and $u_{0}(x)$ is the initial function.

Let us denote

$$
\mathrm{X}_{N}=\left\{v \in P_{N}: v(-1)=v(1)=0\right\} .
$$

The Galerkin method for (1)-(3) is: Find $u_{N} \in P_{N} \cap H_{0}^{1}(\Omega)$ such that:

$$
\left\{\begin{array}{l}
\partial_{t} u_{N}=\varepsilon\left(\partial_{x} u_{N}, \partial_{x} v_{N}\right)+\left(u_{N} \partial_{x} u_{N}, v_{N}\right), \quad v_{N} \in X_{N}, \\
u_{N}\left(x_{i}, 0\right)=u_{0}\left(x_{i}, 0\right), \quad 0 \leq i \leq N .
\end{array}\right.
$$

We consider Chebyshev-Galerkin method in this paper. Let $\left\{x_{j}\right\}_{j=0}^{N}$ be the Chebyshev-Gauss-Lobatto points on the $\Omega, \omega=\left(1-x^{2}\right)^{-1 / 2}$ be the weight function. Given a set of basis functions $\left\{\varphi_{j}\right\}_{j=0}^{N-2}$ of $\mathrm{XN}_{\mathrm{N}}$, we denote

$$
\begin{gathered}
u_{N}=\sum_{j=0}^{N-2} \hat{u}_{j} \varphi_{j}, \quad u=\left(\hat{u}_{0}, \quad \hat{u}_{1}, \cdots, \quad \hat{u}_{N-2}\right)^{T}, \\
S_{k j}=-\int_{I} \omega \varphi_{j}^{\prime \prime} \varphi_{k} d x, \quad m_{k j}=-\int_{I} \omega \varphi_{j} \varphi_{k} d x, \\
S=\left(s_{k j}\right)_{0 \leq k, j \leq N-2}, \quad M=\left(m_{k j}\right)_{0 \leq k, j \leq N-2} .
\end{gathered}
$$

From the paper[7]: if the initial function such that $u_{0}(x) \in H^{m}(\Omega), m \geq 2$, then Euqations (1)-(3) have a unipue solution $\mathrm{u}$ and $\left(\frac{\partial}{\partial t}\right)^{j} u \in C\left(0, T ; H^{m-j}(\Omega)\right), j \geq 0, m-j \geq 0$ and $\mathrm{j}$ is integer. In addition, there exists a constant $C\left(\left\|u_{0}\right\|_{m}\right)$ such that

$$
\left\|\left(\frac{\partial}{\partial t}\right)^{j} u\right\|_{m-j} \leq C\left(\left\|u_{0}\right\|_{m}\right)
$$

\section{Numerical scheme}

In the equations (1)-(3), We use the Crank-Nicolson Leap-frog scheme in time,and the Chebyshev-Galerkin method in space. Let the time step is $\mathrm{dt}, u^{k}(\bullet)$ is the approximate solution of the exact solution $u(\cdot, k d t)$, where $u^{k}$ is the $(\mathrm{N}+1)$-dimension vector, $u^{k}(j)=u^{k}\left(x_{j}\right) \mathrm{n}=1,2, \ldots$, [T/dt]([ ] is rounding),therefore, the equatons (1)-(3) can discrete as following:

$$
\left(\frac{u_{N}^{k+1}-u_{N}^{k-1}}{2 d t}, v_{N}\right)=\varepsilon\left(\partial_{x} \frac{u_{N}^{k+1}+u_{N}^{k-1}}{2 d t}, \partial_{x} v_{N}\right)-\left(u_{N}^{k} \partial_{x} u_{N}^{k}, v_{N}\right) \text {. }
$$

At each time step,one only needs to solve the following problem:

$$
\varepsilon d t\left(\partial_{\chi} \hat{u}_{N}^{k+1}, \partial_{\chi} v_{N}\right)+\left(\hat{u}_{N}^{k+1}, v_{N}\right)=\left(I_{N} \hat{u}_{N}^{k-1}, v_{N}\right)+\frac{d t}{2}\left(\left(u_{N}^{k}\right)^{2}, \partial_{\chi} v_{N}\right) .
$$

Where $\hat{u}_{N}^{k+1}=\frac{1}{2}\left(u_{N}^{k+1}+u_{N}^{k-1}\right)$, and $I_{N}$ is the Legendre-Gauss-Lobatto interpolation operator. Note that the above scheme requires $u_{N}^{0}$ and $u_{N}^{1}$ to start. We let $u_{N}^{0}$ and $u_{N}^{1}$ is the interpolation of initial condition and exact solution.

Taking $v_{N}=\varphi_{j}$, then the above system can be solved by the following maxtric equation:

$$
(\varepsilon d t S+M) \hat{u}=f .
$$


Where $f=\left(f_{k j}\right)_{0 \leq k, j \leq N-2}, f_{k j}=\left(I_{N} u_{N}^{k-1}, v_{N}^{j}\right)+\frac{d t}{2}\left(\left(u_{N}^{k}\right)^{2}, \partial_{x} v_{N}^{j}\right)$.

\section{Numerical example}

In this section,we present below numerical experiment using the Chebyshev-Galerkin method designed in the previous section. All computations are performed with matlab on a personal computer. We consider the following problem:

The exact solution is

$$
\frac{\partial u}{\partial t}=\varepsilon \frac{\partial^{2} u}{\partial x^{2}}-u \frac{\partial u}{\partial x}, \quad \varepsilon>0
$$

$$
u(x, t)=\kappa\left[1-\tanh \left(\frac{\kappa\left(x-\kappa t-x_{c}\right)}{2 \varepsilon}\right)\right],
$$

Let $\varepsilon=0.1, \kappa=0.5, x_{c}=-3, x \in(-5,5), T=12$, and impose the initial value $u(x, 0)$ and boundary conditions $u( \pm 5, t)$ by using the exact solution. The time step size are $d t=10^{-k}, k=2,3,4$, the number of Chebyshev spectral collocation points are32,64,128 at $\mathrm{T}=12$.

In Figure 1, $\mathrm{n}$ is the number of basis function of the Chebyshev-Galerkin method, $d t$ is the time step. We can observe the approximate solutions converge exponentially to the exact solution. In addition, when the time step value is smaller, the Chebyshev- Galerkin method can get the higher accuracy of $10^{-9}$.
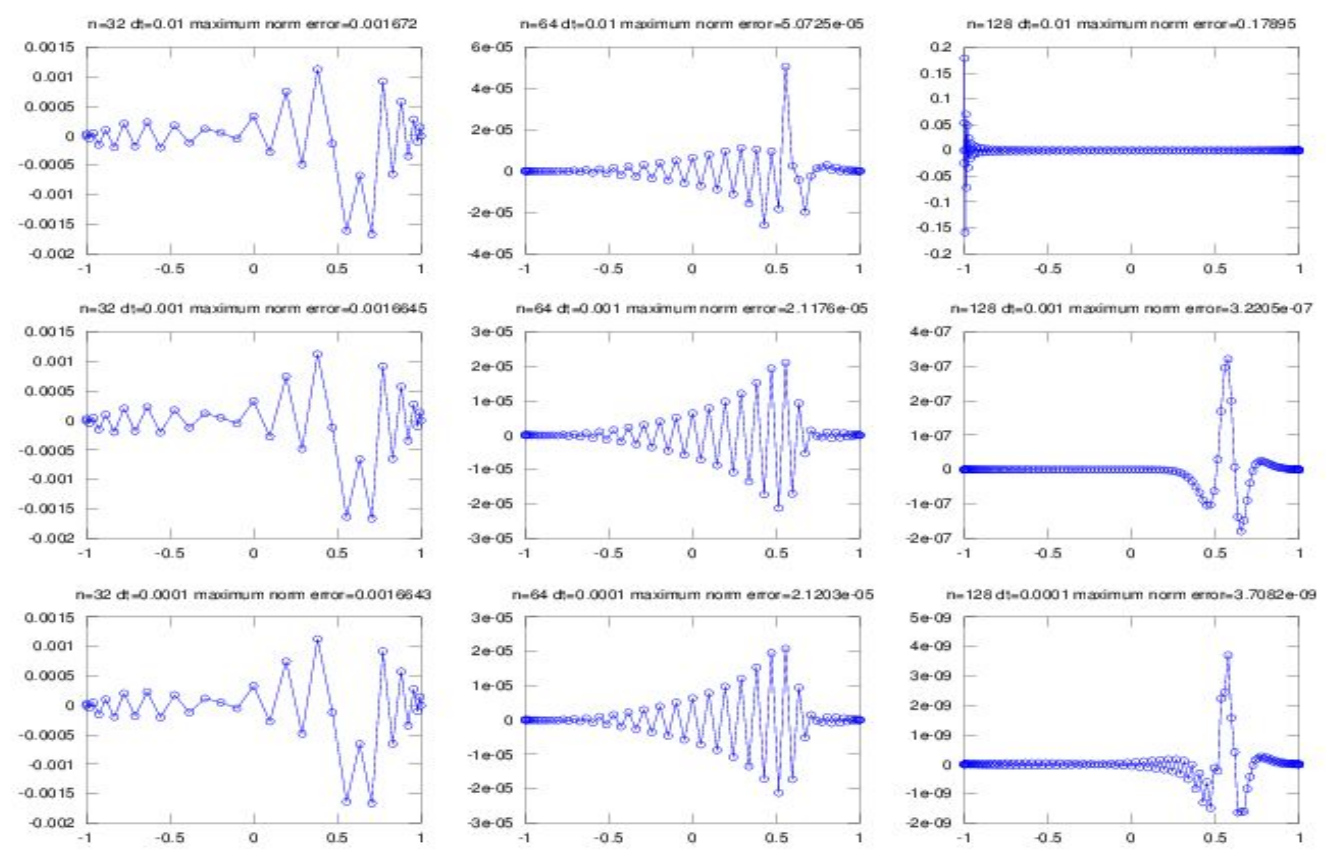

Figure 1: the discrete maximum errors.

\section{Concluding remarks}

We developed an efficient Chebyshev-Galerkin method for nonlinear Burgers equation. There are some advantages of using Chebyshev-Galerkin method, e.g., the condition numbers of the method are all small and independent of $n$. In addition, our method can quickly and effectively calculate the results of high accuracy, this is the other advantage in dealing with nonlinear problems. How to efficiently solve the more complication problems and how to analysis the error will be the further work. 


\section{References}

[1] C A Fletcher. A comparison of finite element and finite difference solution of theone-and two-dimensional Burgers equations[J]J Comp Phys,1983( 51) : 159-188.

[2] J Shen, T Tang. High Order Numerical Methods and Algorithms[M].Beijing: Science press, 2007.

[3] Luo zhendong, Liu ruxun. Mixed finite element analysis and the numerical simulation of Burgers equation[J].computational mathematics,2008,21( 3) : 257-268.

[4] Jin xiaokang,Liu ruxun,Jiang bocheng. Computational Fluid Dynamics[M].changsha: National University of Defense Technology,1994.

[5] Bateman.Some tecent researches on the motion of fluids[J].Monthly Weather Rev,1915 ( 43) : 163-170.

[6] E Benton,G W Platzman.A table of solutions of the one dimensional Burgers equation[J].Quart Appl Math,1972( 30) : 195-212.

[7] Y D Shang.Initial boundary value problem for a class of generalized Burgers type equations[J].Mathematica Applicata,1996( 9) : 166-171.

[8] J Shen. efficient spectral-Galerkin method II. Direct solvers for second- and fourth-order equations by using Chebyshev polynomials[J].SIAM J Sci,Comp, 1995( 16) : 74-87.

[9] Z Battles, L N Trefethen. An extension of matlab to continuous functions and Operators [J].SIAM J Sci Comp,2004( 25) : 1743-1770.

[10] C Canuto, M Y Hussaini, A Quarteroni, T A Zang. Spectral Method sin Fluid Dynamics[M].New York: Springer Verlag,1988.78. 